\title{
Pruebas de transformadores, espectroscopía en el dominio de la frecuencia y método de análisis de respuesta por barrido de frecuencia
}

\author{
Néstor Xavier Maya Izurieta y Luis Alberto Vásquez Restrepo \\ nxavier4@hotmail.com
}

\section{Resumen}

La espectroscopía es una técnica mediante la cual podemos observar gráficamente la interacción entre materia y energía radiada, ya sea en función de la longitud de onda o la frecuencia, lo que nos permite aislar e identificar la estructura constructiva de un material. Esta técnica aplicada a los transformadores nos brinda una herramienta para medir pérdidas y capacitancia en el sistema de aislamiento, en función a la respuesta de frecuencia de una señal aplicada de donde obtenemos la espectroscopía en el dominio de la frecuencia (DFR). El método de análisis de respuesta por barrido de frecuencia SFRA (Sweep Frequency Response Analysis), a su vez, es una técnica de diagnóstico para detectar deformaciones y desplazamientos (entre otras fallas eléctricas y mecánicas) sobre los devanados en transformadores de potencia. En ambos casos la detección del problema se traduce directamente en el tipo de mantenimiento que se debe realizar, considerando altos costos de tiempo y dinero.

Palabras clave: prueba de transformadores, SFRA, respuesta de barrido de frecuencia, DFR, espectroscopía en el dominio de la frecuencia.

\section{Abstract:}

The spectroscopy is a technique by which we can see graphically the interaction between matter and energy radiated, either as a function of wavelength or frequency, allowing us to isolate and identify the structural design of a material. Technique applied to processors gives us a tool to measure losses and capacitance in the insulation, depending on the frequency response of a signal applied spectroscopy from which we get the frequency domain (DFR). The method of analysisof frequency response sweep SFRA (Sweep Frequency Response Analysis) in turn is a diagnostic technique to detect deformations and displacements (includingelectrical and mechanical failures) on the windings in power transformers. In bothcases the detection problem directly translates into the type of maintenance to be performed, considering high costs of time and money.

Keywords: Test of transformers, SFRA, swept frequency response, DFR, spectroscopy in the frequency domain. 


\section{Introducción}

Hace poco tiempo, las opciones para realizar análisis predictivo sobre el estado de un transformador eran muy limitadas. Simplemente se ignoraban ciertos comportamientos, de haberlos, y si realmente había sospechas de daños importantes, se pagaban costosas inspecciones que requerían de mucho tiempo y que no siempre daban respuestas claras. Todo esto ha cambiado gracias a los avances tecnológicos en el campo de la ingeniería eléctrica y electrónica; avances que han creado poderosas herramientas como el análisis de respuesta de barrido de frecuencia y la espectroscopía en el dominio de la frecuencia, que ayuda a controlar el estado y permiten un mejor mantenimiento del transformador.

Estas pruebas permiten realizar una imagen del interior del transformador y detectar incluso cambios sutiles en la estructura mecánica del núcleo, los arrollamientos, la humedad y temperatura en el sistema de aislamiento entre otros factores, sin que esto implique operaciones complicadas o costos extras.

El objetivo principal es detectar desplazamientos una vez producidas sobre corrientes originadas por fallas pasantes, sincronizaciones incorrectas, etcétera. Otras aplicaciones son: la verificación a la condición mecánica luego del transporte del transformador; así como, la detección de cualquier problema que se traduzca en cambios a la distribución de inductancia o capacitancia en transformadores (fallas en el núcleo, problemas en la puesta a tierra del núcleo o pantallas, etcétera).

La comparación con otras técnicas de diagnóstico muestran que las ventajas principales del SFRA y la espectroscopía en el dominio de la frecuencia son su sensibilidad a las distintas fallas que se pueden presentar en arrollamien- tos y sistemas de aislamiento, y una menor dependencia sobre mediciones previas de referencia. Estos fenómenos hacen necesaria una metodología de interpretación sistemática y objetiva.

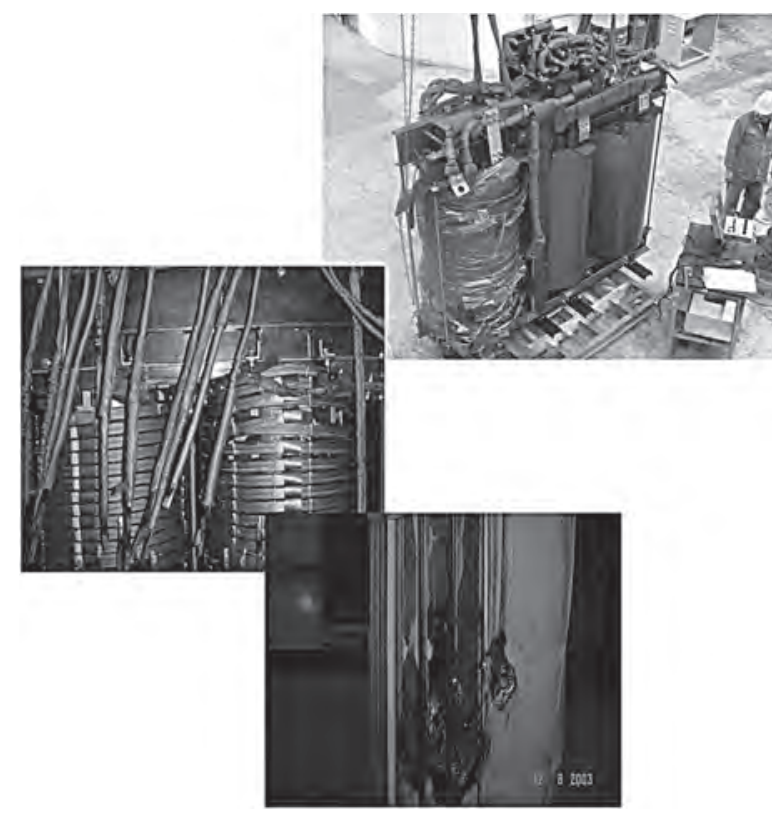

Figura 1.

\section{Espectroscopía en el dominio de la frecuencia (DFR)}

La espectroscopía en el dominio de la frecuencia DFR tiene como base fundamental la interacción entre la materia y la energía radiada al momento de aplicar una señal. Esto permite identificar la estructura química de un compuesto, lo que puede ser aplicado para la detección de un factor de humedad y temperatura dentro de un transformador de potencia.

\subsection{Fundamento teórico}

La espectroscopía en el dominio de la frecuencia utiliza la espectroscopía de la impedancia que trabaja con componentes o valo- 
res complejos en el eje imaginario. En 1941 se introduce en el campo de la respuesta del dieléctrico mediante el gráfico de Cole-Cole.

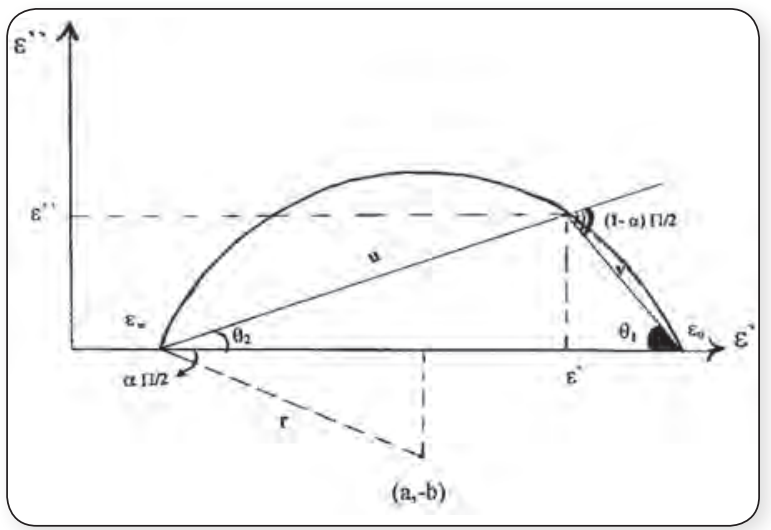

Figura 2.

El modelo de Cole-Cole ha sido utilizado con éxito para describir los datos experimentales de la constante dieléctrica de muchos materiales en función de la frecuencia. En este modelo la constante dieléctrica depende principalmente de cuatro parámetros:

- La constante dieléctrica estática $\varepsilon 0$.

- La constante dieléctrica en el infinito frecuencia $\varepsilon \infty$.

- El tiempo de relajación $\tau$ o $\alpha$ y un exponente de los factores.

En principio, $\varepsilon \circ$ y $\varepsilon \infty$ pueden medirse de forma experimental y los otros dos parámetros o $\tau$ y $\alpha$ tienen que ser tratados como parámetros de ajuste, cuyos valores se pueden recuperar de la mejor ajuste a los datos experimentales.

En la mayoría de los casos, sin embargo, $\varepsilon o$ y $\varepsilon \infty$ no pueden medirse experimentalmente, ya que es dificil medir frecuencias muy bajas y muy altas para detectar la saturación en los valores de los límites.

La parte imaginaria de la permitividad relativa compleja contiene las perdidas resistivas (conductividad DC) y las pérdidas dieléctricas (polarización).

\subsection{La medición en la prueba de espectroscopía en el dominio de la frecuencia (DFR)}

En la prueba DRF se mide la capacitancia y el factor de potencia o factor de disipación en un rango de frecuencia de $1 \mathrm{mHz}$ y $1 \mathrm{Mhz}$.

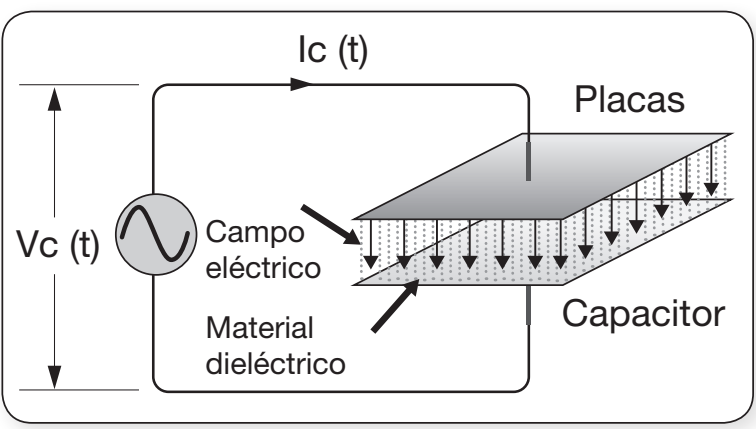

Figura 3.

Aquí las propiedades eléctricas que se deben evaluar son:

- Capacitancias real e imaginaria.

- Factor de disipación o factor de potencia equivalente.

Con esto podemos dar diversas áreas de aplicación donde las correspondientes a nuestra investigación son :

- El aislamiento de los transformadores de potencia.

- Bujes.

- Transformadores de instrumentos. 


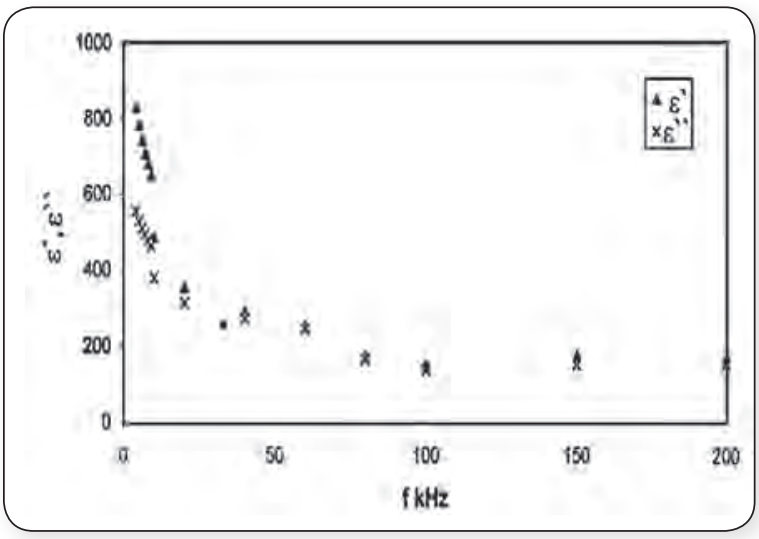

Figura 4.

\subsection{Humedad en trasformadores}

Para realizar una explicación sobre la medición de la humedad en transformadores, es necesario revisar las pruebas comunes que se realizan con este objetivo:

- Muestra de aceite.

- Punto de rocío.

- Unidades de monitoreo en línea.

- Mediciones de factor de potencia.

Cada una de éstas puede resultar en un método invasivo al transformador, lo que nos lleva a buscar una herramienta como la espectroscopía en el dominio de la frecuencia donde podemos obtener una curva de factor de potencia dependiendo de la humedad y temperatura.

Esta curva inicial la podemos comparar con un valor de curva referente obtenida por históricos, y así podemos comparar la respuesta de diferentes aislamientos en función de la frecuencia y el factor de potencia.

Con esto podemos dar una interpretación de la curva en función de la humedad y temperatura que son los factores que pueden modificarla.

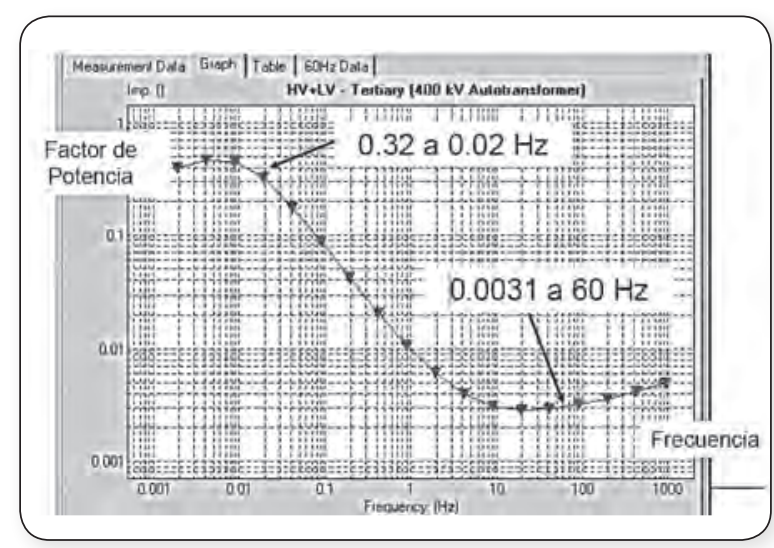

Figura 5.

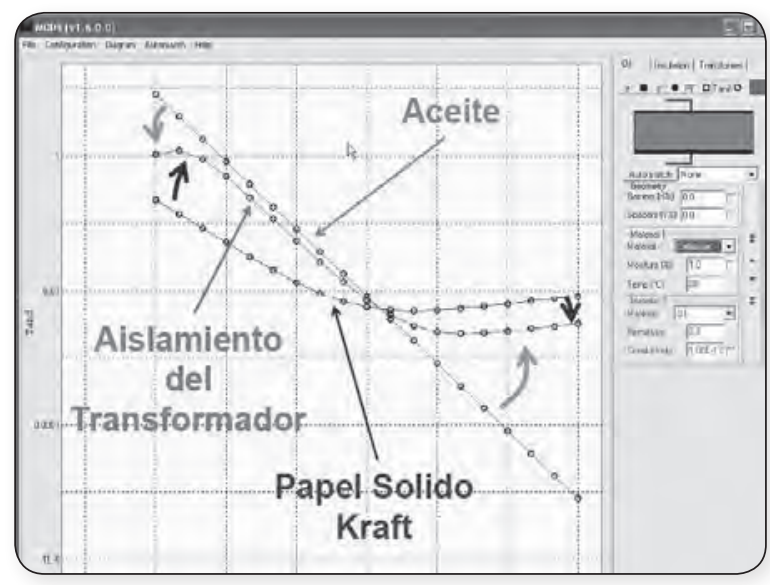

Figura 6.

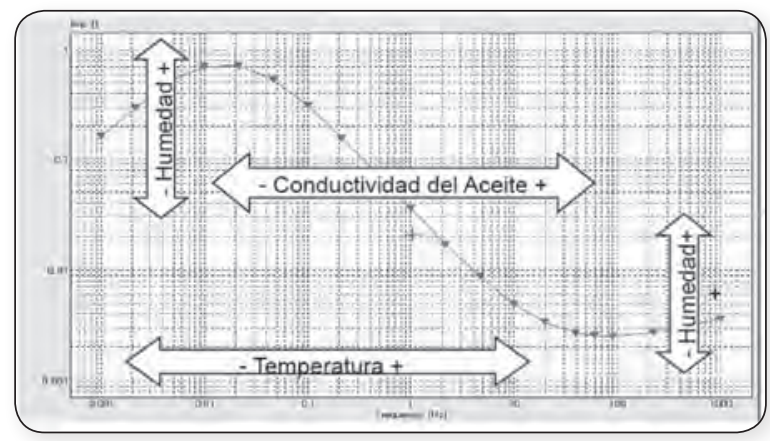

Figura 7.

Así podemos dar un mejor diagnóstico sobre la situación del transformador en el tema de aislamiento que posee. Consecuentemente se puede dar una mejor extensión a la curva de vida de nuestro transformador. 


\section{Análisis de la respuesta de barrido de frecuencia (SFRA)}

SFRA es una técnica probada y estandarizada para realizar mediciones precisas y repetibles.

El dispositivo envía una señal de excitación al transformador midiendo las señales de respuesta, luego compara la respuesta recibida con la del valor basal y otros resultados (por ejemplo, de unidades similares) y así identifica desviaciones y confirma 'problemas mecánicos internos', tales como:

- Movimiento de núcleo.

- Deformación y desplazamiento del arrollamiento.

- Fallas en el núcleo.

- Colapso parcial del arrollamiento.

- Estructuras de sujeción rotas y/o sueltas.

- Cortocircuitos en arrollamientos.

- Verificar la calidad y estado de nuevos transformadores.

- Mejorar la calidad de su programa de inspección regular o mantenimientos.

- Combatir los problemas del sistema, como fallas de cortocircuito que podrían dañar los transformadores.

- Inspeccionar la presencia de daños que haya experimentado el transformador después de terremotos, estrés mecánico, rayos $\mathrm{u}$ otros factores ambientales.

- Eliminar costosas e innecesarias inspecciones internas.

\subsection{Funcionamiento del SFRA}

Una serie de señales se aplican al transformador en diferentes frecuencias.

Se mide la amplitud y la fase de las señales de entrada y salida.

La relación entre estas señales de entrada y salida provee la respuesta de frecuencia o función de transferencia del transformador.

De la función de transferencia se pueden derivar una serie de términos como una función de la frecuencia:

- Magnitud.

- Fase.

- Impedancia/ admitancia.

- Correlación.

El circuito RLC posee una impedancia diferente en cada frecuencia.

La función de transferencia para todas las frecuencias es la medida de la impedancia efectiva del circuito RCL.

Cualquier deformación en la geometría del sistema cambia el circuito RCL, el cual cambia su impedancia y a su vez, su función de transferencia a diferentes frecuencias.

\subsection{Fundamentos de la prueba mediante el SFRA}

Es una prueba que se realiza off line (con el equipo desenergizado).

Se analiza el transformador como un circuito filtro RLC complejo. 


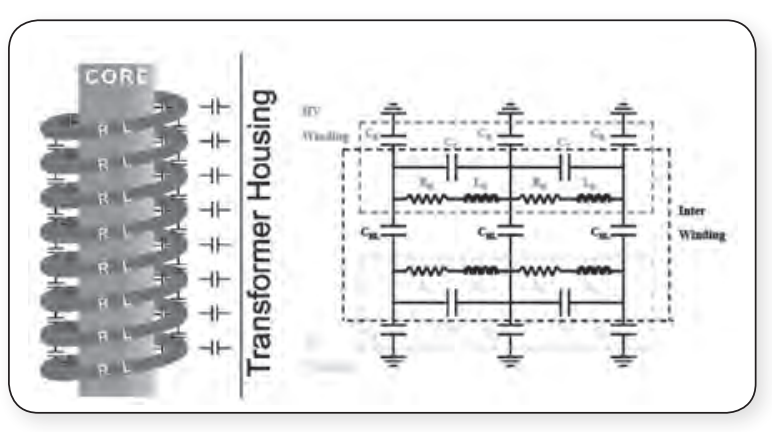

Figura 8.

La respuesta del circuito de filtro se mide en un gran número de frecuencias y se lo grafica como curva de magnitud de respuesta.

Los cambios en el circuito del filtro pueden detectarse mediante comparación en el tiempo.

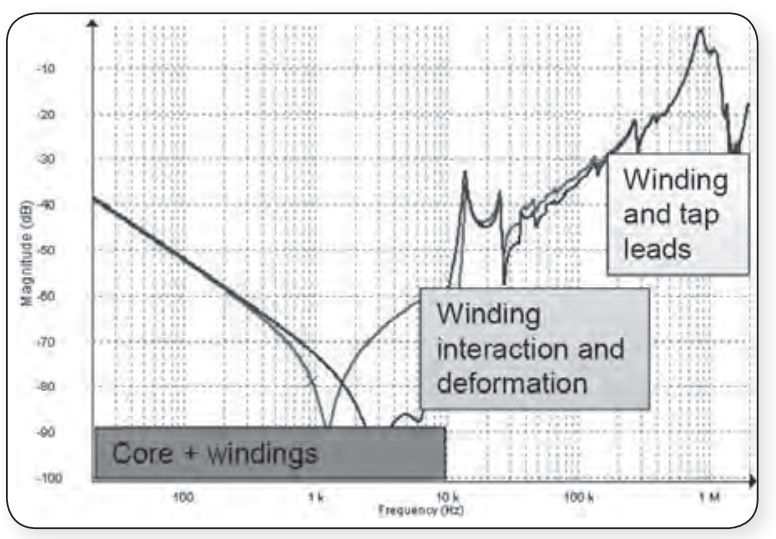

El método es único por la capacidad de detectar variedad de fallas en el núcleo en una sola prueba.

Problemas en el transformador pueden detectarse a diferentes rangos de frecuencias

\section{Bajas frecuencias:}

- Problemas en el núcleo.

- Devanados abiertos /cortocircuito.

- Malas conexiones.

- Cambios en la impedancia de corto circuito.

\section{Medias frecuencias:}

- Deformaciones en los devanados.

- Desplazamiento de los devanados.

\section{Altas frecuencias:}

- Movimiento de devanados y conexionado de conmutador.

Las mediciones y gráficas obtenidas se las compara con mediciones patrones realizadas anteriormente como, por ejemplo, cuando el equipo salió de fábrica o antes de ser energizado.

Figura 9.

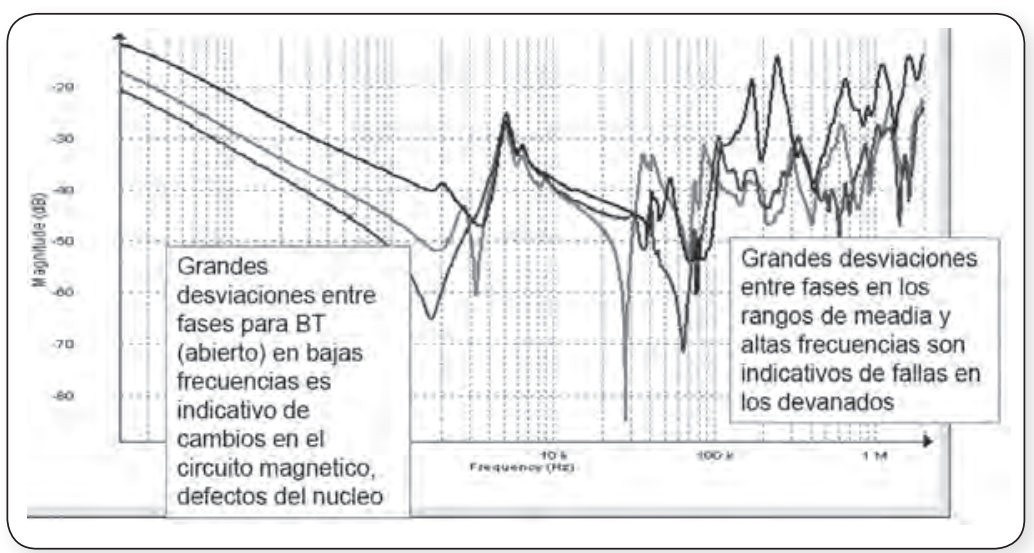

Figura 10 
A estos y más casos podemos dar una clasificación de estas comparaciones:

- Basadas en el tiempo: las pruebas se las realizan al transformador alrededor de su vida, guardando históricos para compararlos con los siguientes valores; es la prueba más eficaz, ya que desviaciones en la curva son fáciles de detectar.

- Basadas en el tipo constructivo: se utilizan patrones de transformadores con diseño similar, se necesita un conocimiento específico sobre el objeto a prueba y sus posibles cambios en el transcurso de su vida, desviaciones menores no son necesariamente signos de problemas en la unidad.

- Basadas en el diseño: las pruebas se las realiza en terminales de los devanados y boquillas de idéntico diseño. Se necesita un conocimiento específico sobre el objeto a prueba y sus posibles cambios en el transcurso de su vida; desviaciones menores no son necesariamente signos de problemas en la unidad.

\section{Regiones de frecuencia según IEEE}

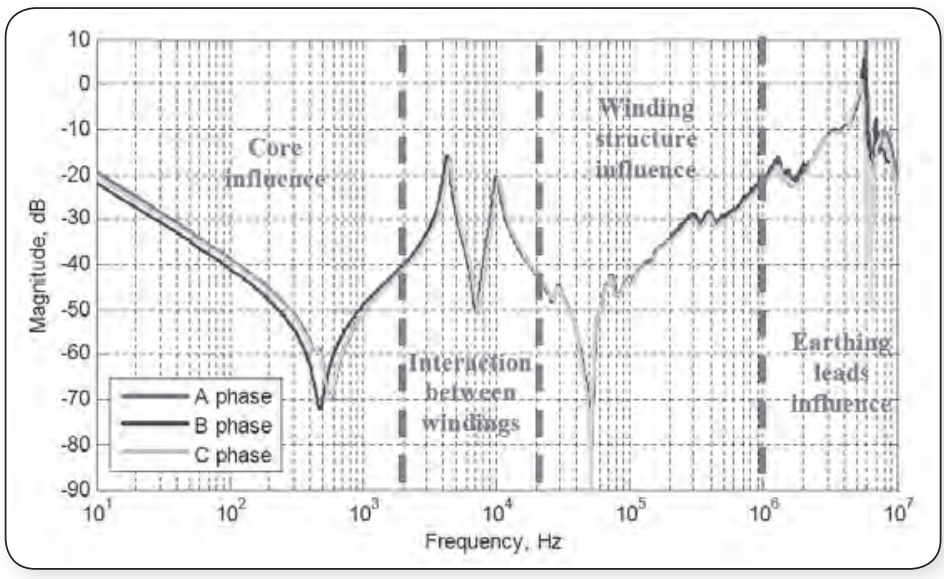

Figura 11

\section{Conclusiones}

- La tecnología no dejará de evolucionar en el proceso de búsqueda de fallas, sobre todo en transformadores de potencia que son el corazón de cualquier red eléctrica.

- La relación de los principios físicos y químicos de la materia, poco a poco, se vinculan a la solución de problemas del sector eléctrico, específicamente en pruebas a realizar para normatividad y mantenimiento.

- La espectroscopía en el dominio de la frecuencia nos da una herramienta para iden- tificar nuevas formas de manejar los datos y posibles causas de fallas antes desconocidas o de fácil acceso, lo que contribuye a los sistemas de mantenimiento y planificación.

- Queda muy clara la filosofía del principio del SFRA, la cual es a nuevas mediciones iguales a mediciones anteriores, el transformador puede operar sin ningún inconveniente, y que a su vez nuevas mediciones diferentes a mediciones anteriores requieren de mayor investigación en el transformador. || ||| 


\section{Referencias bibliográficas}

[1] Samiha T. Bishay, Numerical Methods for the calculations of the Cole-Cole parameters, Phypics department, AimShams University, Cairo, Egypt .

[2] Megger, “BPS 4 _ DFR parte I”, 2011.

[3] Megger, "BPS 6 _ SFRA", 2011. 